\title{
ON THE GROWTH OF MEROMORPHIC FUNCTIONS WITH SEVERAL DEFICIENT VALUES $\left({ }^{1}\right)$
}

\author{
BY \\ ALBERT EDREI AND WOLFGANG H. J. FUCHS()
}

Introduction. Let $f(z)$ be an entire function of finite order $\lambda$ and let $M(r)$ denote its maximum modulus in the region $|z| \leqq r$. The following well known proposition is easy to prove.

Theorem A. If some value $\tau(\neq \infty)$ is exceptional in the sense of Borel, then

(i) $\lambda$ is a positive integer;

(ii) $\log M(r) \cong \alpha r^{\lambda}$ for some positive value of $\alpha$.

In this paper, we investigate the possibility of proving analogous theorems for meromorphic functions possessing deficient values (in the sense of $R$. Nevanlinna).

The main interest of the results obtained lies in the fact that they provide partial answers to the three following questions.

I. Under which conditions are deficiencies invariant under a change of origin?

II. When are deficient values also asymptotic values?

III. How does the presence of deficient values influence the gap structure of the Taylor expansion of an entire function?

We leave aside questions II and III which will be treated in another paper [1].

We explain our notations in $\$ 1$ before stating our results in $\$ 2$.

1. Terminology and notations. The complex variable will be denoted by

$$
z=x+i y=r e^{i \theta} \quad(x, y, \theta \text { real } ; r \geqq 0) .
$$

The function $f(z)$ is, in general, meromorphic.

The sequence of its zeros (other than the origin) will be denoted by

$$
a_{1}, a_{2}, a_{3}, \cdots,
$$

and the sequence of its poles (other than the origin) by

$$
b_{1}, b_{2}, b_{3}, \cdots \text {. }
$$

As usual, the moduli of the terms of these two sequences are taken to be

Received by the editors September 10, 1958.

(1) Research sufported by the United States Air Force, Contract No. AF18(600)-85, monitored by the Office of Scientific Research.

(2) The authors also gratefully acknowledge support by the National Science Foundation and the John Simon Guggenheim Memorial Foundation. 
nondecreasing and each zero or pole appears as often as its multiplicity indicates. By

$$
d_{1}, d_{2}, d_{3}, \cdots,
$$

we denote the sequence obtained by rearranging (1.1) and (1.2) as a single sequence, the terms of which have nondecreasing moduli.

The standard symbols of the Nevanlinna theory

$$
\stackrel{+}{\log ,} M(r, f), m(r, f), n(r, a), N(r, a), N(r, f), T(r, f),
$$

are used throughout the paper; familiarity with their meaning is assumed.

If no confusion is to be feared, we write $M(r), T(r), \cdots$ instead of $M(r, f), T(r, f), \cdots$.

We define

$$
\begin{aligned}
n(r) & =n(r, 0)+n(r, \infty), \\
N(r) & =N(r, 0)+N(r, \infty) .
\end{aligned}
$$

The letters $\lambda$ and $\mu$ denote the order and lower order of $f(z)$, respectively:

$$
\lambda=\limsup _{r \rightarrow \infty} \frac{\log T(r)}{\log r}, \quad \mu=\liminf _{r \rightarrow \infty} \frac{\log T(r)}{\log r} .
$$

We say that $f(z)$ is of regular growth, if $\lambda=\mu$ (both may be $+\infty$ ). The deficiency $\delta(\tau, f)$ of the value $\tau$, with respect to $f(z)$, is, by definition,

$$
\delta(\tau, f)=1-\limsup _{r \rightarrow \infty} \frac{N(r, \tau)}{T(r, f)} .
$$

If no confusion is to be feared, we write $\delta(\tau)$ instead of $\delta(\tau, f)$. An important part is played, in this paper, by the quantity

$$
\kappa=\kappa(f)=\limsup _{r \rightarrow \infty} \frac{N(r)}{T(r)} .
$$

Clearly

$$
\kappa \leqq \limsup \frac{N(r, 0)}{T(r)}+\limsup _{r \rightarrow \infty} \frac{N(r, \infty)}{T(r)}=2-\delta(0)-\delta(\infty) .
$$

2. Statement and discussion of results. Let $\Pi_{\lambda}(z)$ denote the canonical product formed with the sequence of zeros $\left\{a_{\nu}\right\}$ defined by

$$
a_{\nu}=-\nu^{1 / \lambda} \quad(\nu=1,2,3, \cdots, 0<\lambda<+\infty) .
$$

Lindelöf obtained an asymptotic representation of $\Pi_{\lambda}(z)$ which readily yields $[5$, p. 54$]$ : 


$$
\left\{\begin{array}{l}
\kappa\left(\Pi_{\lambda}(z)\right)=1-\delta(0)=\frac{|\sin \pi \lambda|}{q+|\sin \pi \lambda|} \\
\quad\left(q<\lambda \leqq q+\frac{1}{2}, q \geqq 0, \text { integer }\right), \\
\kappa\left(\Pi_{\lambda}(z)\right)=1-\delta(0)=\frac{|\sin \pi \lambda|}{q+1} \\
\left(q+\frac{1}{2}<\lambda \leqq q+1, q \geqq 0, \text { integer }\right) .
\end{array}\right.
$$

It is therefore clear that the assertion (i) of Theorem A cannot hold for all meromorphic functions satisfying the inequality

$$
\kappa(f)<1 .
$$

However, the following Theorem B, of R. Nevanlinna, shows that some connection exists between the order $\lambda$, of $f(z)$, and the numerical value of $\kappa(f)$.

Theorem B. Let

$$
k(\lambda)=\inf (\kappa(f))
$$

where $f$ ranges over all meromorphic functions of order $\lambda$. Then

$$
k(\lambda)=0
$$$$
(\lambda=1,2,3, \cdots) .
$$

For all other $\lambda, k(\lambda)>0$.

$\mathrm{R}$. Nevanlinna posed the problem of determining the exact value of $k(\lambda)$. Using an important lemma of A. A. Goldberg [3], we have obtained a complete solution of this problem for $\lambda<1$. A detailed account of our work in this direction will appear elsewhere [2].

In the general case $\lambda<+\infty$, which will be considered here, our results are not as precise. We prove

THEOREM 1. Let $f(z)$ be a meromorphic function of finite order $\lambda$. Then

$$
\kappa(f) \geqq \frac{|\sin \pi \lambda|}{2.2 \lambda+\frac{1}{2}|\sin \pi \lambda|} .
$$

This result gives the correct order of magnitude of $k(\lambda)$ since (2.1), (2.3) and (2.4) yield

$$
\kappa\left(\Pi_{\lambda}(z)\right) \geqq k(\lambda) \geqq \frac{|\sin \pi \lambda|}{2.2 \lambda+\frac{1}{2}|\sin \pi \lambda|} \geqq \frac{1}{3.3} \kappa\left(\Pi_{\lambda}(z)\right) \quad(\lambda \geqq 1) .
$$


(For $\lambda<1,(2.4)$ is superseded by the result to appear in [2].)

Next we turn to generalizations of the second part of Theorem $A$.

First we shall show that an entire function may well have a finite deficient value without being of regular growth. This is easily deduced from the following theorem, which is also of independent interest.

THEоRем 2. Let $f(z)$ be an entire function vanishing at all points of the sequence $\left\{a_{v}\right\}_{\nu=1}^{\infty}$ and nowhere else. Assume that

(i) $a_{\nu}<0(\nu=1,2,3, \cdots)$;

(ii) $\sum\left|a_{\nu}\right|^{-\rho}$ converges for some finite value of $\rho$;

(iii) $\sum\left|a_{v}\right|^{-1}$ diverges.

Then

$$
\delta(0, f)>\frac{A}{1+\log q}
$$$$
(q \geqq 1),
$$

where $A$ is an absolute constant and $q$ is the genus of the canonical product formed with the zeros $a_{\nu}$.

Canonical products of finite genus, with negative zeros, need not be of regular growth. It is easy to see that, for a suitable sequence $\left\{a_{\nu}\right\}$, with occasional very large gaps between consecutive terms, $\lambda=q+1, \mu=q$. Let $g(z)$ be such a product; then, by Theorem $2, \delta(0, g)>0$, provided $q \geqq 1$. Moreover, taking $q=1$ and replacing $z$ by $z^{k}$, we obtain examples of entire functions, possessing deficient zeros, and such that $\lambda=2 k, \mu=k(k=1,2,3, \cdots)$.

It might be of interest to mention, without proof, that it is possible to construct, for arbitrarily small positive values of $\epsilon$, entire functions with $\lambda=1-\epsilon, \mu=1 / 2-\epsilon, \delta(0)>0$.

A study of these examples suggests the following problem which we are unable to solve:

If $f(z)$ is entire and of finite order $\lambda$, does the presence of a finite deficient value imply $\lambda \leqq 2 \mu$ ?

Theorem 2 raises another interesting question:

Which sequences $\left\{a_{v}\right\}$ have the property that, if an entire function $f(z)$ vanishes at all points $a_{\nu}$, and nowhere else, then $\delta(0, f)>0$ ?

Theorem 2 disproves the conjecture that such sequences are associated with functions $n(r, 0)$ possessing special properties (that is other properties than the obvious properties of all counting-functions).

Although Theorem 2 shows that an inequality such as (2.2) has little influence on the regularity of the growth of the characteristic of a meromorphic function, a closer inspection reveals that the assertion (ii) of Theorem A, may be generalized if $\kappa$ is sufficiently small (Theorems 4 and 5). This generalization is basic in our results concerning the problems mentioned in the Introduction. Its proof is based on the two following companion theorems.

THEOREM 3a. Let $f(z)$ be meromorphic. If $\sigma>1$ and $r>2$, then 
(2.5) $T(r) \leqq \frac{4}{\sigma-1} T(\sigma r)+\max \{N(\sigma r, 0), N(\sigma r, \infty)\}+O(\log r)(r \rightarrow \infty)$. If $f(z)$ is entire,

$$
\log M(r) \leqq \frac{4}{\sigma-1} T(\sigma r)+N(\sigma r, 0)+O(\log r) \quad(r \rightarrow \infty) .
$$

Theorem 3b. Let $q$ be a non-negative integer. Put

$$
\alpha=e^{1 /(q+1)},
$$

and let $E(u, q)$ denote the primary factor of genus $q$.

If $f(z)$ is a meromorphic function with $f(0)=1$, then for

$$
0<2 \rho<r<\frac{1}{2} R
$$

we have

$$
\begin{aligned}
\log \left|f\left(r e^{i \theta}\right)\right| & =\log |f(z)| \\
& =\sum_{p<\left|a_{\nu}\right| \leq R} \log \left|E\left(\frac{z}{a_{\nu}}, q\right)\right|-\sum_{p<\left|b_{\nu}\right| \leq R} \log \left|E\left(\frac{z}{b_{\nu}}, q\right)\right|+S,
\end{aligned}
$$

where

(2.9) $\left\{\begin{aligned}|S| \leqq 2 N(\alpha \rho)+N(r) & +\frac{r}{R}\{8 T(\alpha R)+2 N(\alpha R)\} & (q=0), \\ |S| \leqq\left(\frac{r}{\rho}\right)^{q}\{8 T(\alpha \rho) & \left.+\frac{5(q+1)}{q} N(\alpha \rho)\right\} & \\ & +\left(\frac{r}{R}\right)^{q+1}\{8 T(\alpha R)+2 N(\alpha R)\} & (q \geqq 1) .\end{aligned}\right.$

Further, if

$$
\phi(t)=\frac{1}{\pi} \int_{0}^{\pi} \frac{d \theta}{\left(t^{2}-2 t \cos \theta+1\right)^{1 / 2}}
$$

then

$$
\begin{aligned}
2 T(r)-N(r)< & (q+1) r^{q} \int_{\rho}^{R} N(\alpha t) t^{-q-1} \phi\left(\frac{t}{r}\right) d t \\
& +2 N(\alpha R)\left(\frac{r}{R}\right)^{q+1}+|S|
\end{aligned}
$$

where $|S|$ satisfies (2.9). 
From Theorem 3a, we deduce

THEOREM 4. Let $f(z)$ be meromorphic and assume that two of its values $\alpha, \beta$ are deficient. If

$$
\gamma=\max \{1-\delta(\alpha), 1-\delta(\beta)\}
$$

then

$$
\begin{array}{ll}
\mu \geqq \frac{\log \left(\frac{1}{\gamma(2-\gamma)}\right)}{\log \left(1+\frac{4}{\gamma(1-\gamma)}\right)} \quad(\gamma \neq 0), \\
\mu \geqq 1 & (\gamma=0) .
\end{array}
$$

CoRollary 4.1. Meromorphic functions with more than one deficient value have a positive lower order.

From Theorem $3 b$, we deduce

THEOREM 5. Let $f(z)$ be a meromorphic function. Assume that for some nonnegative integer $q$ and some $\beta, 0<\beta \leqq 1 / 2$,

$$
\kappa(f)<\frac{\beta}{5 e(q+1)} .
$$

I. If

$$
\lambda>q+1-\beta
$$

then every interval

$$
x \leqq r \leqq(35)^{1 / \beta} x
$$

contains a point s such that

$$
T(u) u^{-q-1+\beta} \leqq T(s) s^{-q-1+\beta} \quad\left(x_{0} \leqq u \leqq s\right) .
$$

II. If

$$
u<q+\beta,
$$

then every interval (2.13) contains a point $t$ such that

$$
T(t) t^{-q-\beta} \geqq T(v) v^{-q-\beta}
$$

Corollary 5.1. If (2.11) and (2.12) hold, then

$$
\mu \geqq q+1-\beta .
$$

If (2.11) and (2.14) hold, then 


$$
\lambda \leqq q+\beta .
$$

Combined with Theorems 1 and 4, Corollary 5.1 yields

THEOREM 6. Let $f(z)$ be a meromorphic function of finite lower order $\mu$. Let $p$ be the integer defined by

$$
p-\frac{1}{2} \leqq \mu<p+\frac{1}{2} .
$$

If

$$
\kappa(f)<\frac{\beta}{5 e(p+1)}
$$$$
\left(0<\beta \leqq \frac{1}{2}\right)
$$

then $p \geqq 1$,

$$
|\lambda-p|<\frac{\beta}{10}
$$

and

$$
p-\beta \leqq \mu \leqq \lambda<p+\frac{\beta}{10} .
$$

As an immediate consequence, we obtain the following generalization of Theorem A.

CoROLlaRy 6.1. If $\kappa(f)=0$, then the order of $f(z)$ is either infinite or a positive integer. In both cases, $f(z)$ is of regular growth.

Valiron [10] proved

THEOREM C. If $f(z)$ is of finite order $\lambda$, and if $\lambda-\mu<1$, then all deficiencies are invariant under a change of origin.

Combined with Theorem 6, this gives

COROLLARY 6.2. If $f(z)$ is a meromorphic function of lower order $\mu$ and if

$$
\kappa(f)<\frac{1}{10 e\left(\mu+\frac{3}{2}\right)},
$$

then all deficiencies are invariant under a change of origin.

It is interesting to combine some of our results with the following

LEMma 1. Let $f(z)$ be a meromorphic function of finite order. If

$$
\Delta=\sum_{r \rightarrow \infty} \delta(\tau, f)>1-\gamma \quad(0<\gamma<1),
$$


and

$$
\delta(\infty, f)>1-\gamma
$$

then

$$
1-\gamma<\liminf _{r \rightarrow \infty} \frac{T\left(r, f^{\prime}\right)}{T(r, f)} \leqq \limsup _{r \rightarrow \infty} \frac{T\left(r, f^{\prime}\right)}{T(r, f)}<1+\gamma,
$$

and

$$
\kappa\left(f^{\prime}\right) \leqq \frac{4 \gamma}{1-\gamma^{2}} .
$$

In the special case of entire functions, it is clear that the condition (2.17) may be omitted. Lemma 1 then shows that if $\Delta$ is sufficiently close to 1 , $\kappa\left(f^{\prime}\right)$ will be so small that some of our results may be applied to $f^{\prime}$. This yields information about $T\left(r, f^{\prime}\right)$ which, in view of (2.18), may be expressed in terms of $T(r, f)$.

Combining Corollary 6.1 and Lemma 1, we thus obtain

ThEOREM 7. An entire function of finite order with

$$
\sum_{r} \delta(\tau)=2 \text {, }
$$

is necessarily of positive integral order and of regular growth.

Using Lemma 1, in the same way, it is clearly possible to restate other results of this paper. The modified theorems will be applicable to entire functions with $\Delta$ close to one.

Using the full strength of Lemma 1, there will be further extensions to meromorphic functions with $\sum \delta(\tau)$ close to two and one deficient value of deficiency close to one.

It would be interesting to omit the latter restriction. We are unable to do this, but observe that such an omission would necessarily weaken some of our statements. It is known, for instance, that part of Theorem 7 does not hold for all meromorphic functions satisfying the condition (2.20), since there exist functions of this type and of finite nonintegral order $[4$, p. 83].

3. Estimates for the logarithmic mean of the primary factor of genus $q$. Let $q(\geqq 0)$ be an integer and put

$$
\left\{\begin{array}{l}
E(u, 0)=1-u, \\
E(u, q)=(1-u) \exp \left(u+\frac{u^{2}}{2}+\cdots+\frac{u^{q}}{q}\right) \quad(q>0) .
\end{array}\right.
$$

Since $q$ is fixed throughout this section, we write $E(u)$ instead of $E(u, q)$. We start from the representation 


$$
\log E(u)=\int_{0}^{u} \frac{\xi^{q}}{\xi-1} d \xi,
$$

where the path of integration can be chosen as a straight line segment, provided $u$ is not a positive number greater or equal to 1 . Hence, for $0<\theta<2 \pi$,

$$
|\log | E\left(r e^{i \theta}\right)|| \leqq \int_{0}^{r} \frac{t^{a} d t}{\left|t e^{i \theta}-1\right|} .
$$

Integrating with respect to $\theta$ gives

$$
\text { (3.2) } \frac{1}{2 \pi} \int_{0}^{2 \pi}|\log | E\left(r e^{i \theta}\right)|| d \theta=m(r, E(z))+m\left(r, \frac{1}{E(z)}\right)=\int_{0}^{r} t^{q} \phi(t) d t
$$

where

$$
\phi(t)=\frac{1}{2 \pi} \int_{0}^{2 \pi} \frac{d \theta}{\left|t e^{i \theta}-1\right|}=\frac{1}{2 \pi} \int_{0}^{2 \pi} \frac{d \theta}{\left(t^{2}-2 t \cos \theta+1\right)^{1 / 2}} .
$$

The function $\phi(t)$ is defined for all positive $t(\neq 1)$, and it is easy to verify that

$$
\begin{aligned}
\phi(t) & =\frac{1}{1+t}\left\{1+\sum_{m=1}^{\infty}\left(\frac{1 \cdot 3 \cdot 5 \cdots(2 m-1)}{2 \cdot 4 \cdot 6 \cdots 2 m}\right)^{2}\left(\frac{4 t}{(1+t)^{2}}\right)^{m}\right\} \\
& \leqq \frac{1}{1+t}\left\{1+\frac{2}{\pi} \log \left|\frac{1+t}{1-t}\right|\right\} .
\end{aligned}
$$

We shall require several properties of $\phi(t)$, in particular

$$
t \phi(t)=\phi\left(\frac{1}{t}\right)
$$

which readily follows from (3.3). For $t \geqq 2$,

$$
0<\phi(t)<\frac{1}{2 \pi} \int_{0}^{2 \pi} \frac{d \theta}{t-1}<\frac{2}{t} .
$$

By (3.2) and (3.5),

(3.7) $m(r, E(z))+m\left(r, \frac{1}{E(z)}\right) \leqq \int_{1 / r}^{\infty} t^{-q-1} \phi(t) d t=r^{q} \int_{1}^{\infty} u^{-q-1} \phi\left(\frac{u}{r}\right) d u$.

We now evaluate the integral

$$
\int_{0}^{\infty} t^{\beta-1} \phi(t) d t=J(\beta)
$$

which, in view of (3.6), is convergent for $0<\beta<1$. Clearly, 


$$
J(\beta)=\frac{1}{\pi} \int_{0}^{\pi} d \theta \int_{0}^{\infty} \frac{t^{\beta-1}}{\left(t^{2}-2 t \cos \theta+1\right)^{1 / 2}} d t,
$$

and the change of variable

$$
t=\cos \theta+\sin \theta \tan \phi=\frac{\cos (\theta-\phi)}{\cos \phi},
$$

transforms (3.8) into

$$
J(\beta)=\frac{1}{\pi} \int_{0}^{\pi} d \theta \int_{\theta-\pi / 2}^{\pi / 2}\{\cos (\theta-\phi)\}^{\beta-1}\{\cos \phi\}^{-\beta} d \phi .
$$

Interchanging again the order of integration

$$
J(\beta)=\frac{1}{\pi} \int_{-\pi / 2}^{\pi / 2}(\cos \phi)^{-\beta} d \phi \int_{0}^{\phi+\pi / 2}\{\cos (\theta-\phi)\}^{\beta-1} d \theta .
$$

Now

(3.10) $\int_{0}^{\phi+\pi / 2}\{\cos (\theta-\phi)\}^{\beta-1} d \theta=\int_{0}^{\pi / 2}\{\cos \omega\}^{\beta-1} d \omega+\int_{-\phi}^{0}\{\cos \omega\}^{\beta-1} d \omega$, where the latter integral is an odd function of $\phi$. Hence (3.9) and (3.10) yield

$$
J(\beta)=\frac{1}{\pi} \int_{-\pi / 2}^{\pi / 2}\{\cos \phi\}^{-\beta} d \phi \int_{0}^{\pi / 2}\{\cos \omega\}^{\beta-1} d \omega .
$$

Expressing these integrals in terms of the $\Gamma$-function $[11$, p. 256], we find

$$
J(\beta)=\frac{\Gamma^{2}\left(\frac{1}{2}\right) \Gamma\left(\frac{\beta}{2}\right) \Gamma\left(\frac{1-\beta}{2}\right)}{2 \pi \Gamma\left(1-\frac{\beta}{2}\right) \Gamma\left(\frac{1}{2}+\frac{\beta}{2}\right)} .
$$

Using the identity

$$
\Gamma(z) \Gamma(1-z)=\frac{\pi}{\sin \pi z}
$$

we obtain

$$
J(\beta)=\frac{\pi^{2}}{\sin (\pi \beta) \Gamma^{2}\left(\frac{3}{4}+\frac{1-2 \beta}{4}\right) \Gamma^{2}\left(\frac{3}{4}-\frac{1-2 \beta}{4}\right)} .
$$

The factor of $\sin (\pi \beta)$ in the denominator has a minimum at $\beta=1 / 2$. This is readily seen by examining 


$$
\frac{d}{d t} \log \Gamma\left(\frac{3}{4}+t\right) \Gamma\left(\frac{3}{4}-t\right)=\sum_{m=0}^{\infty} \frac{2 t}{\left(\frac{3}{4}+m\right)^{2}-t^{2}}
$$

For $|t|<1 / 4$, the left-hand side of (3.12) has the sign of $t$, so that $\Gamma(3 / 4+t) \Gamma(3 / 4-t)$ has a minimum at $t=0$. Hence, by $(3.11)$

$$
\int_{0}^{\infty} t^{\beta-1} \phi(t) d t=J(\beta) \leqq \frac{\pi^{2}}{\Gamma^{4}(3 / 4) \sin (\pi \beta)}<\frac{4.4}{\sin \pi \beta} .
$$

4. Proof of Theorem 1. Let $f(z)$ be a meromorphic function of finite nonintegral order $\lambda$. Then $f(z)$ has the canonical representation

$$
f(z)=z^{k} e^{P(z)} \frac{\prod E\left(\frac{z}{a}\right)}{\prod E\left(\frac{z}{b}\right)} \quad(E(u)=E(u, q) ; q=[\lambda]),
$$

where $E(u)$ is defined by (3.1) and $P(z)$ is a polynomial of degree not greater than $q$.

We write $\left\{d_{\nu}\right\}$ for the sequence obtained by rearranging the zeros $\left\{a_{\nu}\right\}$ and the poles $\left\{b_{\nu}\right\}$ of $f(z)$ in a single sequence $\left(0<\left|d_{1}\right| \leqq\left|d_{2}\right| \leqq\left|d_{3}\right| \cdots\right)$. Obviously

$$
\sum_{|d,| \leq t} 1=n(t, 0)+n(t, \infty)=n(t) .
$$

From (4.1), we deduce

$$
|\log | f(z)|| \leqq \sum_{v=1}^{\infty}|\log | E\left(\frac{z}{d_{v}}\right)||+O\left(r^{q}\right) \quad(r \rightarrow+\infty),
$$

and integrating with respect to $\theta\left(z=r e^{i \theta}\right)$, we find

$$
m(r, f)+m\left(r, \frac{1}{f}\right) \leqq \sum_{r=1}^{\infty}\left\{m\left(r, E\left(\frac{z}{d_{v}}\right)\right)+m\left(r, \frac{1}{E\left(z / d_{v}\right)}\right)\right\}+O\left(r^{q}\right) .
$$

By (3.7)

$$
\begin{aligned}
m\left(r, E\left(\frac{z}{d}\right)\right)+m & \left(r, \frac{1}{E(z / d)}\right)=m\left(\frac{r}{|d|}, E(z)\right)+m\left(\frac{r}{|d|}, \frac{1}{E(z)}\right) \\
& \leqq\left(\frac{r}{|d|}\right)^{q} \int_{1}^{\infty} u^{-q-1} \phi\left(\frac{u|d|}{r}\right) d u=r^{q} \int_{|d|}^{\infty} t^{-q-1} \phi\left(\frac{t}{r}\right) d t .
\end{aligned}
$$

Therefore 


$$
m(r, f)+m\left(r, \frac{1}{f}\right) \leqq r^{q} \sum_{v=1}^{\infty} \int_{|d v|}^{\infty} t^{-q-1} \phi\left(\frac{t}{r}\right) d t+O\left(r^{q}\right) .
$$

Since

$$
\sum_{r=1}^{\infty} \int_{|d, p|}^{\infty} t^{-q-1} \phi\left(\frac{t}{r}\right) d t=\int_{0}^{\infty} n(t) t^{-q-1} \phi\left(\frac{t}{r}\right) d t
$$

and

$$
T(r)=m(r, f)+N(r, \infty)=m\left(r, \frac{1}{f}\right)+N(r, 0)+O(\log r),
$$

(4.2) implies

$$
2 T(r)-N(r) \leqq r^{q} \int_{0}^{\infty} n(t) t^{q-1} \phi\left(\frac{t}{r}\right) d t+O\left(r^{q}\right) \quad(r \rightarrow+\infty) .
$$

We choose $\epsilon(>0)$ such that

$$
q+\epsilon<\lambda<q+1-\epsilon .
$$

By (4.3) and the definition of $\kappa(f)$ there is a constant $C$ such that, for $r>r_{0}$,

$$
(2-\kappa-\epsilon) T(r) \leqq r^{q} \int_{0}^{\infty} n(t) t^{-q-1} \phi\left(\frac{t}{r}\right) d t+C r^{q} .
$$

Choosing $\boldsymbol{\gamma}$ such that

$$
\lambda<\boldsymbol{\gamma}<\boldsymbol{\lambda}+\epsilon,
$$

multiplying (4.5) by $r^{-\gamma-1}$ and integrating from $x$ to $+\infty$, we obtain

$$
\begin{aligned}
(2-\kappa-\epsilon) \int_{x}^{\infty} T(r) r^{-\gamma-1} d r \leqq & \int_{x}^{\infty} r^{q-\gamma-1} d r \int_{0}^{\infty} n(t) t^{-q-1} \phi\left(\frac{t}{r}\right) d t \\
& +C \frac{x^{q-\gamma}}{\gamma-q}
\end{aligned}
$$

Denote by $I$ the repeated integral in (4.7); interchanging the order of the integrations, we find

$$
I=\int_{0}^{\infty} n(t) t^{-q-1} d t \int_{z}^{\infty} r^{q-\gamma-1} \phi\left(\frac{t}{r}\right) d r .
$$

The substitution $r=t s$ yields

$$
I=\int_{0}^{\infty} n(t) t-1-1 d t \int_{s / t}^{\infty} s^{q-\gamma-1} \phi\left(\frac{1}{s}\right) d s
$$


We now set

$$
\begin{aligned}
& G(t)=\int_{t}^{\infty} s^{q-\gamma-1} \phi\left(\frac{1}{s}\right) d s \\
& F(t)=\int_{t}^{\infty} n(s) s^{-\gamma-1} d s=-N(t) t^{-\gamma}+\gamma \int_{t}^{\infty} N(s) s^{-\gamma-1} d s,
\end{aligned}
$$

so that (4.8) takes the form

$$
I=-\int_{0}^{\infty} G\left(\frac{x}{t}\right) d F(t)
$$

An integration by parts yields

$$
I=\int_{0}^{\infty} F(t)\left(\frac{x}{t}\right)^{q-\gamma} \phi\left(\frac{t}{x}\right) \frac{d t}{t} .
$$

Clearly

$$
t^{\epsilon} F(t) \geqq n(t) t^{\epsilon} \int_{t}^{\infty} \frac{d s}{s^{\gamma+1}}=\frac{n(t)}{\gamma t^{\gamma-\epsilon}},
$$

and since $n(t)$ is of order $\lambda$, the second of the inequalities (4.6) yields

$$
\limsup _{t \rightarrow \infty} t^{e} F(t)=+\infty \text {. }
$$

Hence there must exist an increasing, unbounded sequence $\left\{x_{\nu}\right\}$ such that, for each $x_{v}$,

$$
i F(t) \leqq x_{v}^{c} F\left(x_{v}\right) \quad\left(t \leqq x_{v}\right) .
$$

On the other hand, by definition, $F(t)$ is a nonincreasing function so that

$$
F(t) \leqq F\left(x_{v}\right)
$$$$
\left(t \geqq x_{v}\right) \text {. }
$$

Using (4.12) and (4.13), in (4.10), we obtain for $x=x$,

$$
\begin{aligned}
I & \leqq F\left(x_{v}\right)\left\{\int_{0}^{x_{\nu}}\left(\frac{x_{v}}{t}\right)^{q-\gamma+\epsilon} \phi\left(\frac{t}{x_{v}}\right) \frac{d t}{t}+\int_{x_{\nu}}^{\infty}\left(\frac{x_{v}}{t}\right)^{q-\gamma} \phi\left(\frac{t}{x_{v}}\right) \frac{d t}{t}\right\} \\
& =F\left(x_{v}\right)\left\{\int_{0}^{1} u^{\gamma-q-1-\epsilon} \phi(u) d u+\int_{1}^{\infty} u^{\gamma-q-1} \phi(u) d u\right\} \\
& =A(\epsilon) F\left(x_{v}\right) .
\end{aligned}
$$

We now consider (4.7) with $x=x_{\nu}$ and use the estimate (4.14) for the repeated integral:

(4.15) $\quad(2-\kappa-\epsilon) \int_{x_{\nu}}^{\infty} T(r) r^{-\gamma-1} d r \leqq F\left(x_{\nu}\right)\left\{A(\epsilon)+\frac{C x_{\nu}^{q-\gamma}}{(\gamma-q) F\left(x_{\nu}\right)}\right\}$. 
1959] MEROMORPHIC FUNCTIONS WITH SEVERAL DEFICIENT VALUES 305

In view of (4.11) and (4.12), for sufficiently large $\nu$

$$
\frac{1}{F\left(x_{v}\right)} \leqq x_{v}
$$

and hence

$$
\lim _{\nu \rightarrow \infty} \frac{x_{\nu}^{q-\gamma}}{F\left(x_{\nu}\right)}=0
$$

since

$$
q-\gamma+\epsilon<0
$$

by (4.4) and (4.6).

Combining (4.15) and (4.16), we obtain

$$
\frac{2-\kappa-\epsilon}{A(\epsilon)} \leqq \limsup _{x \rightarrow \infty} \frac{F(x)}{\int_{x}^{\infty} T(r) r^{-r-1} d r} .
$$

By (4.9) and the definition of $\kappa(f)$, we also have

(4.18) $\quad \limsup _{x \rightarrow \infty} \frac{F(x)}{\int_{x}^{\infty} T(r) r^{-r-1} d r} \leqq \limsup _{x \rightarrow \infty} \frac{\gamma \int_{x}^{\infty} N(r) r^{-\gamma-1} d r}{\int_{x}^{\infty} T(r) r^{-\gamma-1} d r} \leqq \gamma \kappa(f)$,

so that (4.17) and (4.18) yield

$$
\frac{2-\kappa-\epsilon}{\gamma A(\epsilon)} \leqq \kappa=\kappa(f) .
$$

Now let $\epsilon \rightarrow 0$; then $\gamma \rightarrow \lambda$ and

$$
A(\epsilon) \rightarrow \int_{0}^{\infty} u^{\lambda-q-1} \phi(u) d u .
$$

Hence

$$
\frac{2-\kappa}{\lambda \int_{0}^{\infty} u^{\lambda-q-1} \phi(u) d u} \leqq \kappa=\kappa(f)
$$

Theorem 1 follows by solving this inequality for $\kappa$ and using the estimate (3.13). 
5. A property of canonical products with negative zeros. We prove the following:

Lemma 2. Let $g(z)$ be a canonical product of finite genus $q(\geqq 1)$. If all the zeros of $g(z)$ are real and negative, then

$$
\delta(0, g)>\frac{A}{1+\log q},
$$

where $A$ is an absolute constant.

Proof. We start from the well-known representation

$$
\log g(z)=(-1)^{q} \int_{0}^{\infty} n(x) \frac{z^{q+1}}{x^{q+1}(z+x)} d x,
$$

due to Valiron [8, p. 237]. Taking real parts,

(5.1) $\log \left|g\left(r e^{i \theta}\right)\right|=(-1)^{q_{r}^{q+1}} \int_{0}^{\infty} \frac{n(x)}{x^{q+1}} \frac{x \cos (q+1) \theta+r \cos q \theta}{x^{2}+r^{2}+2 x r \cos \theta} d x$.

We first assume that $q$ is odd and consider the $[q / 4]+1$ arcs defined by (5.2) $\frac{2 \pi k}{q+1}+\frac{\pi}{4(q+1)} \leqq \theta \leqq \frac{2 \pi k}{q+1}+\frac{\pi}{3(q+1)} \quad(k=0,1,2, \ldots,[q / 4])$.

Their total length is greater than $\pi q / 48(q+1)$, and on each of these arcs

$$
\cos (q+1) \theta \geqq \frac{1}{2} .
$$

Now (5.2) also implies

$$
2 \pi k+\frac{\pi}{4} \frac{q-8 k}{q+1} \leqq q \theta<2 \pi k+\frac{\pi}{3} \quad(k=0,1,2, \cdots,[q / 4]),
$$

and since $q-8 k \geqq-q$, we have

$$
\cos q \theta \geqq \frac{1}{2} .
$$

In view of (5.1), (5.3) and (5.4)

$$
\log \left|\frac{1}{g\left(r e^{i \theta}\right)}\right| \geqq \frac{r^{q+1}}{2} \int_{0}^{\infty} \frac{n(x)}{x^{q+1}} \frac{x+r}{x^{2}+r^{2}+2 x r} d x
$$

on arcs of total length greater than $\pi / 100$. Hence

$$
m\left(r, \frac{1}{g}\right) \geqq \frac{r^{q+1}}{400} \int_{0}^{\infty} \frac{n(x)}{x^{q+1}} \frac{d x}{x+r} .
$$


Using the estimates (3.2) and (3.4), it is easy to see that there exists an absolute, positive constant $B$, such that

$$
m(r, E(z, q))+m\left(r, \frac{1}{E(z, q)}\right) \leqq B(1+\log q) \int_{0}^{r} \frac{t^{q}}{1+t} d t .
$$

This implies

$$
m\left(r, E\left(\frac{z}{a_{r}}, q\right)\right)+m\left(r, \frac{1}{E\left(\frac{z}{a_{v}}, q\right)}\right) \leqq B(1+\log q) \int_{\left|a_{\mid}\right|}^{\infty}\left(\frac{r}{x}\right)^{q+1} \frac{d x}{x+r}
$$

$$
T(r, g(z)) \leqq B(1+\log q) r^{q+1} \int_{0}^{\infty} \frac{n(x)}{n^{q+1}} \frac{d x}{x+r},
$$

since $g(z)$ is a canonical product.

Comparing (5.5) and (5.6), we obtain the lemma for odd values of $q$.

If $q(>0)$ is even, we would consider the $[(q-2) / 6]+1$ arcs defined by

$$
\frac{2 \pi k}{q+1}+\frac{\pi}{q+1} \leqq \theta \leqq \frac{2 \pi k}{q+1}+\frac{5 \pi}{4(q+1)}\left(k=0,1,2, \cdots,\left[\frac{q-2}{6}\right]\right) .
$$

Their total length is still bounded from below, independently of $q$, and on these arcs

$$
2 \pi k+\pi \frac{q-2 k}{q+1} \leqq q \theta<2 \pi k+\frac{5}{4} \pi .
$$

Since $k \leqq[(q-2) / 6]$ implies

$$
\frac{q-2 k}{q+1} \geqq \frac{2}{3},
$$

we now have

$$
-\cos q \theta \geqq \frac{1}{2}, \quad-\cos (q+1) \theta \geqq \frac{1}{2} .
$$

From this point on, the proof given for odd values of $q$ applies without modifications.

If $q=0$, the inequalities (5.7) become meaningless because of the restrictions imposed on $k$; it is also clear that, in this case, the lemma is no longer true.

6. Proof of Theorem 2. Let $g(z)$ be the canonical product, of genus $q$, formed with the zeros $a_{r}$.

By definition, $q$ is the smallest integer such that 


$$
\sum \frac{1}{\left|a_{v}\right|^{a+1}}<+\infty,
$$

and hence, $q$ is also the largest integer such that

$$
\sum \frac{1}{\left|a_{v}\right|^{q}}=+\infty
$$

By assumption (iii) of Theorem $2, q \geqq 1$ and by assumption (ii), $q<+\infty$. Hence the results of $\$ 5$ may be applied to $g(z)$; in particular, the inequalities (5.5) and (5.6) yield

$$
C_{r^{q+1}} \int_{0}^{\infty} \frac{n(x)}{x^{q+1}} \frac{d x}{x+r} \leqq T(r, g) \leqq D r^{q+1} \int_{0}^{\infty} \frac{n(x)}{x^{q+1}} \frac{d x}{x+r},
$$

where $C$ and $D$ are suitable positive constants.

It is well known $[9$, pp. 51-52] that (6.1) and (6.2) imply

$$
\int_{0}^{\infty} \frac{n(x)}{x^{q+2}} d x<+\infty
$$

and

$$
\int_{0}^{\infty} \frac{n(x)}{x^{q+1}} d x=+\infty .
$$

Let $\epsilon(>0)$ be given. By (6.4), we may choose $t(>0)$ so that

$$
\int_{t}^{\infty} \frac{n(x)}{x^{q+2}} d x<\epsilon
$$

and hence, by the second inequality (6.3),

$$
T(r, g) \leqq D r^{q+1}\left\{\int_{0}^{t} \frac{n(x)}{x^{q+1}} \frac{d x}{r}+\epsilon\right\}<2 \epsilon D r^{q+1},
$$

provided $r$ is large enough. This clearly yields

$$
\lim _{r \rightarrow \infty} \frac{r^{q+1}}{T(r, g)}=+\infty \text {. }
$$

Using the first inequality (6.3),

$$
C_{r^{q+1}} \int_{0}^{r} \frac{n(x)}{x^{q+1}} \frac{d x}{2 r} \leqq T(r, g) .
$$

and by (6.5)

$$
\lim _{r \rightarrow \infty} \frac{r^{q}}{T(r, g)}=c .
$$


Now consider the most general entire function $f(z)$, with the sequence of zeros $\left\{a_{\boldsymbol{n}}\right\}$ :

$$
f(z)=e^{Q(z)} g(z) \text {. }
$$

If $Q(z)$ is a polynomial of degree at most equal to $q$,

$$
T(r, g) \leqq T(r, f)+T\left(r, e^{-Q}\right) \leqq T(r, f)+H r^{q},
$$

the latter inequality holds for some suitable constant $H$ and all sufficiently large values of $r$. In view of (6.7)

$$
1 \leqq \liminf _{r \rightarrow \infty} \frac{T(r, f)}{T(r, g)},
$$

and this clearly implies

$$
\delta(0, f) \geqq \delta(0, g)>\frac{A}{1+\log q},
$$

by Lemma 2 .

If $Q(z)$ is a polynomial of degree greater than $q$, or an entire function, we use

$$
T\left(r, e^{\ell}\right) \leqq T(r, f)+T(r, g),
$$

instead of (6.8).

If $Q(z)$ is a polynomial of degree greater than $q$

$$
T\left(r, e^{Q}\right)>B r^{q+1},
$$

for some suitable $H$ and $r$ large enough.

If $Q(z)$ is an entire function, we observe that

$$
\log M\left(r, e^{Q}\right)=\max _{|z|=r} R Q(z)
$$

and obtain (6.10) by an obvious argument involving the inequality of BorelCarathéodory, Liouville's theorem and Nevanlinna's inequality relating the logarithm of the maximum modulus and $m\left(r, e^{\boldsymbol{Q}}\right)$.

Now (6.6), (6.9) and (6.10) imply

$$
\lim _{r \rightarrow \infty} \frac{T(r, f)}{T(r, g)}=+\infty,
$$

and hence

$$
\delta(0, f)=1 \text {. }
$$

7. Proof of Theorem 3a. We may suppose, without loss of generality, that $f(z)$ has no zero or poles on $|z|=\sigma r$. (The general case follows from this by an obvious continuity argument.) 
We may also suppose that $f(0)=1$, since

$$
T(r, f)=T\left(r, A z^{\beta} f(z)\right)+O(\log r) .
$$

We start from the Poisson-Jensen formula

$$
\begin{aligned}
\log |f(z)|= & \frac{1}{2 \pi} \int_{0}^{2 \pi} \log \left|f\left(R e^{i \phi}\right)\right| \frac{R^{2}-r^{2}}{R^{2}-2 R r \cos (\theta-\phi)+r^{2}} d \phi \\
& +\sum_{\left|a_{y}\right|<R} \log \left|g\left(z, a_{v}\right)\right|-\sum_{\left|b_{v}\right|<R} \log \left|g\left(z, b_{v}\right)\right|,
\end{aligned}
$$

where

$$
R=\sigma r
$$

and

$$
g(z, \alpha)=\frac{R(z-\alpha)}{R^{2}-\alpha z}
$$

Let

$$
\frac{R^{2}-r^{2}}{R^{2}-2 r R \cos (\theta-\phi)+r^{2}}=1+Q
$$

Then

$$
\begin{aligned}
& \frac{R-r}{R+r} \leqq 1+Q \leqq \frac{R+r}{R-r}, \\
& |Q| \leqq \frac{2 r}{R-r} .
\end{aligned}
$$

Using (7.2) and (7.3) in (7.1) and noting that

$$
|g(z, \alpha)|<1 \quad(|z|<R),
$$

we obtain

$$
\text { (7.4) } \begin{aligned}
\log |f(z)| \leqq & \frac{1}{2 \pi} \int_{0}^{2 \pi} \log \left|f\left(R e^{i \phi}\right)\right| d \phi+\frac{2}{\sigma-1} \int_{0}^{2 \pi}|\log | f\left(R e^{i \phi}\right)|| d \phi \\
& -\sum_{\left|b_{v}\right|<R} \log \left|g\left(z, b_{v}\right)\right| .
\end{aligned}
$$

Now

(7.5) $\frac{1}{2 \pi} \int_{0}^{2 \pi} \log \left|f\left(R e^{i \phi}\right)\right| d \phi=N(R, 0)-N(R, \infty) \quad$ (Jensen's formula), and 
(7.6) $\frac{1}{2 \pi} \int_{0}^{2 \pi}|\log | f\left(R e^{i \phi}\right)|| d \phi=m(R, f)+m\left(R, \frac{1}{f}\right) \leqq 2 T(R)$.

Combining (7.4), (7.5) and (7.6) we obtain

(7.7) $\log ^{+}|f(z)| \leqq\left(N(R, 0)-N(R, \infty)^{+}+\frac{4 T(R)}{\sigma-1}-\sum_{\left|b_{y}\right|<R} \log \left|g\left(z, b_{v}\right)\right|\right.$, where $X^{+}$denotes $\max \{0, X\}$.

If $f(z)$ is entire, it is clear that (7.7) is equivalent to

$$
\log M(r) \leqq \frac{4}{\sigma-1} T(\sigma r)+N(\sigma r, 0) \quad(R=\sigma r) .
$$

If $f(z)$ is meromorphic, we integrate (7.7) with respect to $\theta$ and notice that, by Jensen's formula,

$$
-\sum_{\left|b_{r}\right|<R} \frac{1}{2 \pi} \int_{0}^{2 \pi} \log \left|g\left(r e^{i \theta}, b_{r}\right)\right| d \theta=N(R, \infty)-N(r, \infty) .
$$

We thus obtain

$$
\begin{aligned}
T(r) & =m(r, f)+N(r, \infty) \\
& \leqq \frac{4}{\sigma-1} T(\sigma r)+(N(\sigma r, 0)-N(\sigma r, \infty))++N(\sigma r, \infty) \\
& =\frac{4}{\sigma-1} T(\sigma r)+\max \{N(\sigma r, 0), N(\sigma r, \infty)\} .
\end{aligned}
$$

The inequalities (7.9) and (7.8) coincide, respectively, with (2.5) and (2.6) except for the $O(\log r)$ term which appears if the condition $f(0)=1$ is dropped.

8. Proof of Theorem 3b. Let $f(z)$ be meromorphic, $f(0)=1$. Consider the polynomials

$$
\Pi_{0}(z)=\prod_{\left|a_{v}\right| \leq R}\left(1-\frac{z}{a_{v}}\right), \quad \Pi_{\infty}(z)=\prod_{\left|b_{v}\right| \leq R}\left(1-\frac{z}{b_{v}}\right),
$$

formed, respectively, with the zeros and poles of $f(z)$. The function

$$
h(z)=\log \left\{\frac{\Pi_{\infty}(z)}{\Pi_{0}(z)} f(z)\right\}
$$

is clearly regular for $|z| \leqq R$ and, for a suitable choice of the determination of the logarithm

$$
h(z)=\sum_{m=1}^{\infty} C_{m}(R) z^{m} \quad(|z| \leqq R) .
$$


Since $R$ is fixed throughout this section, we write $C_{m}$ instead of $C_{m}(R)$. To estimate the coefficients $C_{m}$ we use the well known formulae $[6$, p. 86]

$$
C_{m} r^{m}=\frac{1}{\pi} \int_{-\pi}^{+\pi} R\left\{h\left(r e^{i \theta}\right)\right\} e^{-i m \theta} d \theta \quad(r \leqq R, m \geqq 1) .
$$

If $0<|d|<|z|=r$,

$$
\Re \log \left(1-\frac{z}{d}\right)=\log \frac{r}{|d|}-\Re \sum_{m=1}^{\infty} \frac{1}{m} \frac{\bar{d}^{m}}{r^{2 m}} z^{m},
$$

and the formulae (8.3) may be applied to the power series on the right-hand side of (8.4); we thus obtain

$$
-\frac{1}{m} \frac{\bar{d}^{m}}{r^{m}}=\frac{1}{\pi} \int_{-\pi}^{+\pi} \log \left|1-\frac{r e^{i \theta}}{d}\right| e^{-i m \theta} d \theta \quad(0<|d|<r ; m \geqq 1) .
$$

Similarly

$$
-\frac{1}{m} \frac{r^{m}}{d^{m}}=\frac{1}{\pi} \int_{-\pi}^{+\pi} \log \left|1-\frac{r e^{i \theta}}{d}\right| e^{-i m \theta} d \theta \quad(r<|d| ; m \geqq 1) .
$$

By a simple continuity argument, it is easy to see that (8.5) and (8.6) remain valid for $|d|=r$.

Combining (8.3), (8.5) and (8.6), we obtain

$$
C_{m} r^{m}=\frac{1}{\pi} \int_{-\pi}^{+\pi} \log \left|f\left(r e^{i \theta}\right)\right| e^{-i m \theta} d \theta+\frac{1}{m} \sum_{\left|a_{\nu}\right| \leq r} \frac{\bar{a}_{\nu}^{m}}{r^{m}}
$$

$$
+\frac{1}{m} \sum_{r<\left|a_{\nu}\right| \leq R} \frac{r^{m}}{a_{\nu}^{m}}-\frac{1}{m} \sum_{\left|b_{\nu}\right| \leq r} \frac{b_{\nu}^{m}}{r^{m}}-\sum_{r<\left|b_{\nu}\right| \leq R} \frac{r^{m}}{b_{\nu}^{m}} \quad(r \leqq R) .
$$

Now

$$
\left|\frac{1}{\pi} \int_{-\pi}^{+\pi} \log \right| f\left(r e^{i \theta}\right)\left|e^{-i m \theta} d \theta\right| \leqq \frac{1}{\pi} \int_{-\pi}^{+\pi}|\log | f\left(r e^{i \theta}\right)|| d \theta
$$

$$
\begin{aligned}
& =2\left(m(r, f)+m\left(r, \frac{1}{f}\right)\right) \\
& =4 T(r)-2 N(r)
\end{aligned}
$$

so that (8.7) and (8.8) yield

$$
C_{m}=\frac{1}{m} \sum_{r<\left|a_{\nu}\right| \leq R} \frac{1}{a_{\nu}^{m}}-\frac{1}{m} \sum_{r<\left|b_{\nu}\right| \leq R} \frac{1}{b_{\nu}^{m}}+\gamma_{m}(r)
$$

where 


$$
\left|\gamma_{m}(r)\right| \leqq \frac{4 T(r)-2 N(r)}{r^{m}}+\frac{n(r)}{m r^{m}} .
$$

In particular, $r=R$ implies

$$
C_{m}=\gamma_{m}(R)
$$

and hence

$$
\left|C_{m}\right| \leqq \frac{4 T(R)}{R^{m}}+\frac{n(R)}{m R^{m}}
$$

We next choose a bound $\rho(0<\rho<(R / 4))$ and assume

$$
2 \rho<r=|z|<\frac{R}{2} \text {. }
$$

Let $q(\geqq 1)$ be an integer and let $E(u, q)$ denote the primary factor of genus $q$, defined by (3.1). find

Replacing, in (8.9), $r$ by $\rho$, and returning to the definition of $E(u, q)$, we

$$
\begin{aligned}
R\left\{\sum_{m=1}^{q} C_{m} z^{q}-\log \right. & \left.\frac{\prod_{\infty}(z)}{\prod_{0}(z)}\right\}=\sum_{\rho<\left|a_{\nu}\right| \leq R} \log \left|E\left(\frac{z}{a_{\nu}}, q\right)\right| \\
& -\sum_{\rho<\left|b_{\nu}\right| \leq R} \log \left|E\left(\frac{z}{b_{\nu}}, q\right)\right|+\log \left|\prod_{\left|a_{\nu}\right| \leqq \rho}\left(1-\frac{z}{a_{\nu}}\right)\right| \\
& -\log \left|\prod_{\left|b_{\nu}\right| \leq \rho}\left(1-\frac{z}{b_{\nu}}\right)\right|+\propto \sum_{m=1}^{q} \gamma_{m}(\rho) z^{m} .
\end{aligned}
$$

Combining (8.1), (8.2) and (8.13), we obtain

(8.14) $\log |f(z)|=\sum_{\rho<\left|a_{\nu}\right| \leq R} \log \left|E\left(\frac{z}{a_{\nu}}, q\right)\right|-\sum_{\rho<\left|b_{\nu}\right| \leq R} \log \left|E\left(\frac{z}{b_{\nu}}, q\right)\right|+S$, where

$$
\begin{aligned}
S=Q\left\{\sum_{m=1}^{q} \gamma_{m}(\rho) z^{m}+\sum_{m=q+1}^{\infty} C_{m} z^{m}+\log \prod_{\left|a_{\nu}\right| \leq \rho}(\right. & \left(-\frac{z}{a_{\nu}}\right) \\
& \left.-\log \prod_{\left|b_{\nu}\right| \leq \rho}\left(1-\frac{z}{b_{\nu}}\right)\right\} .
\end{aligned}
$$

We now estimate the various terms on the right-hand side of (8.15). We first prove 
$(8.16)$

$$
\sum_{m=1}^{q}\left|\gamma_{m}(\rho)\right| r^{m}<\left\{8 T(\rho)-4 N(\rho)+\frac{4}{q} n(\rho)\right\}\left(\frac{r}{\rho}\right)^{q} .
$$

By (8.10)

$$
\sum_{m=1}^{q}\left|\gamma_{m}(\rho)\right| r^{m} \leqq\{4 T(\rho)-2 N(\rho)\} \sum_{m=1}^{q}\left(\frac{r}{\rho}\right)^{m}+n(\rho) \sum_{m=1}^{q} \frac{1}{m}\left(\frac{r}{\rho}\right)^{m},
$$

and since $(\rho / r)<(1 / 2)$ (by $(8.12))$,

$$
\begin{aligned}
\sum_{m=1}^{q}\left(\frac{r}{\rho}\right)^{m} & <\left(\frac{r}{\rho}\right)^{q} \sum_{m=0}^{\infty}\left(\frac{\rho}{r}\right)^{m}<2\left(\frac{r}{\rho}\right)^{q} ; \\
\sum_{m=1}^{q} \frac{1}{m}\left(\frac{r}{\rho}\right)^{m} & =\frac{1}{q}\left(\frac{r}{\rho}\right)^{q} \sum_{l=0}^{q-1} \frac{q}{q-l}\left(\frac{\rho}{r}\right)^{l} \\
& <\frac{1}{q}\left(\frac{r}{\rho}\right)^{q} \sum_{l=0}^{\infty}(l+1)\left(\frac{\rho}{r}\right)^{l} \leqq \frac{4}{q}\left(\frac{r}{\rho}\right)^{q} .
\end{aligned}
$$

The inequality (8.16) follows from (8.17), (8.18) and (8.19).

Similarly, using (8.11) and (8.12)

$$
\sum_{m=q+1}^{\infty}\left|C_{m}\right| r^{m} \leqq\left\{8 T(R)+\frac{2}{q+1} n(R)\right\}\left(\frac{r}{R}\right)^{q+1} .
$$

We next observe that

$$
|\log | \prod_{\left|a_{\nu}\right| \leq \rho}\left(1-\frac{z}{a_{\nu}}\right)|| \leqq \sum_{\left|a_{\nu}\right| \leq \rho} \log \frac{r}{\left|a_{\nu}\right|}+|\log | \prod_{\left|a_{\nu}\right| \leq \rho}\left(1-\frac{a_{\nu}}{z}\right)||,
$$

and since

$$
1-\frac{\rho}{r} \leqq\left|1-\frac{a_{v}}{z}\right| \leqq 1+\frac{\rho}{r}
$$

we also have

$$
|\log | 1-\frac{a_{\nu}}{z}|| \leqq-\log \left(1-\frac{\rho}{r}\right) .
$$

Hence (8.21) implies

$$
\begin{aligned}
|\log | \prod_{\left|a_{\nu}\right| \leqq \rho}\left(1-\frac{z}{a_{v}}\right)|| & \\
& \leqq N(\rho, 0)+n(\rho, 0)\left\{\log \left(\frac{r}{\rho}\right)-\log \left(1-\frac{\rho}{r}\right)\right\} .
\end{aligned}
$$

By (8.12) 


$$
\frac{r}{\rho}>2, \quad r-\rho>\rho,
$$

so that

$$
\begin{aligned}
\log \left(\frac{r}{\rho}\right)-\log \left(1-\frac{\rho}{r}\right) & =\log \left\{\frac{r^{2}}{\rho(r-\rho)}\right\}<2 \log \frac{r}{\rho}<\frac{r}{\rho} \\
& \leqq \frac{1}{q}\left(\frac{r}{\rho}\right)^{q} \quad(q \geqq 1) .
\end{aligned}
$$

Using (8.23) in (8.22), we obtain

$$
|\log | \prod_{\left|a_{\nu}\right| \leq \rho}\left(1-\frac{z}{a_{v}}\right)|| \leqq N(\rho, 0)+\frac{n(\rho, 0)}{q}\left(\frac{r}{\rho}\right)^{q},
$$

and this estimate remains valid if the zeros $a$ are replaced by the poles $b$.

Combining (8.15), (8.16), (8.20) and (8.24), we finally obtain

$$
|S| \leqq\left\{8 T(\rho)+\frac{5 n(\rho)}{q}\right\}\left(\frac{r}{\rho}\right)^{q}+\left\{8 T(R)+\frac{2 n(R)}{q+1}\right\}\left(\frac{r}{R}\right)^{q+1}
$$

In the above estimate, we have assumed $q \geqq 1$, but it is clearly possible to obtain for $\log |f(z)|$ an expression such as (8.14) with $q=0$. Starting from (8.1) and (8.2), we obtain immediately (8.14) with $q=0$ and

(8.26) $S=R\left\{\sum_{m=1}^{\infty} C_{m} z^{m}+\log \prod_{\left|a_{\nu}\right| \leq \rho}\left(1-\frac{z}{a_{\nu}}\right)-\log \prod_{\left|b_{\nu}\right| \leq \rho}\left(1-\frac{z}{b_{\nu}}\right)\right\}$.

The inequality (8.20) is still valid with $q=0$. There is nothing to change in (8.22) and, by (8.23), its right-hand side may be replaced by

$$
N(\rho, 0)+n(\rho, 0)\left\{1+\log \frac{r}{\rho}\right\} \text {. }
$$

Hence (8.26) yields

$$
|S| \leqq\{8 T(R)+2 n(R)\} \frac{r}{R}+N(\rho)+n(\rho)\left\{1+\log \frac{r}{\rho}\right\} .
$$

Using the fact that $T(u)$ and $N(u)$ are increasing functions and that

$$
n(\rho) \log \frac{r}{\rho} \leqq \int_{\rho}^{r} \frac{n(t)}{t} d t \leqq N(r)
$$

$$
n(u)=n(u)(q+1) \int_{u}^{\alpha u} \frac{d t}{t} \leqq(q+1) N(\alpha u) \quad\left(\alpha=e^{1 /(q+1)}\right),
$$

we obtain (2.8) and (2.9) from (8.14), (8.25) and (8.27). 
From (2.8), we deduce

$$
|\log | f(z)|| \leqq \sum_{\rho<\left|d_{y}\right| \leq R}|\log | E\left(\frac{z}{d_{v}}, q\right)||+|S|,
$$

where $\left\{d_{\nu}\right\}$ denotes again the sequence obtained by combining the sequences $\left\{a_{v}\right\}$ and $\left\{b_{v}\right\}$. Taking mean values, as in the proof of Theorem 1, we find $m(r, f)+m\left(r, \frac{1}{f}\right) \leqq \sum_{p<\left|d_{v}\right| \leq R}\left\{m\left(r, E\left(\frac{z}{d_{v}}\right), q\right)+m\left(r, \frac{1}{E\left(\frac{z}{d_{v}}, q\right)}\right)\right\}+|S|$.

Minor modifications of the arguments which lead to (4.3), now yield $2 T(r)-N(r)$

$$
\leqq r^{q} \int_{\rho}^{R} n(t) t^{-q-1} \phi\left(\frac{t}{r}\right) d t+r^{q} n(R) \int_{R}^{\infty} t^{-q-1} \phi\left(\frac{t}{\dot{r}}\right) d t+|S| .
$$

By (3.6) and (8.28)

$$
\begin{aligned}
r^{q} n(R) \int_{R}^{\infty} t^{-q-1} \phi\left(\frac{t}{r}\right) d t & \leqq 2 r^{q+1} n(R) \int_{R}^{\infty} t^{-q-2} d t \\
& \leqq 2 N(\alpha R)\left(\frac{r}{R}\right)^{q+1} .
\end{aligned}
$$

Inequality (2.10) follows from (8.28), (8.29) and (8.30); this completes the proof of Theorem $3 \mathrm{~b}$.

9. Proof of Theorem 4. We may suppose, without loss of generality, that

$$
\alpha=0, \quad \beta=\infty,
$$

since, by Nevanlinna's first fundamental theorem, a bilinear transformation of $f(z)$ does not alter the lower order of $T(r)$.

Let

$$
\gamma<c^{\prime}<c<1
$$

then the definition of deficiency implies

$$
N(r, 0)<c^{\prime} T(r), \quad N(r, \infty)<c^{\prime} T(r) .
$$

for all sufficiently large values of $r$.

In (2.5), choose

$$
\sigma=1+\frac{4}{c(1-c)} .
$$

Then, for all sufficiently large values of $r$. 


$$
T(r)<T(\sigma r)\left\{\frac{4}{\sigma-1}+c^{\prime}+\frac{A \log r}{T(r)}\right\},
$$

where $A$ is a suitable constant.

Since $f(z)$ is clearly not a rational function,

$$
\frac{\log r}{T(r)} \rightarrow 0
$$

as $r \rightarrow+\infty$, and hence

$$
c^{\prime}+\frac{A \log r}{T(r)}<c,
$$

provided $r$ is sufficiently large.

By (9.1), (9.2) and (9.3), there exists some $r_{0}$ such that

$$
\frac{T\left(\sigma^{k} r_{0}\right)}{T\left(\sigma^{k-1} r_{0}\right)}>\frac{1}{c(2-c)} \quad(k=1,2,3, \cdots) .
$$

This implies

$$
\frac{T\left(\sigma^{n} r_{0}\right)}{T\left(r_{0}\right)}=\prod_{k=1}^{n} \frac{T\left(\sigma^{k} r_{0}\right)}{T\left(\sigma^{k-1} r_{0}\right)}>\left\{\frac{1}{c(2-c)}\right\}^{n} .
$$

Hence, if $r>r_{0}$ and $n$ is determined by

$$
\sigma^{n} r_{0} \leqq r<\sigma^{n+1} r_{0}
$$

we have

$$
\begin{gathered}
\frac{\log T(r)}{\log r}>\frac{\log T\left(\sigma^{n} r_{0}\right)}{\log \left(\sigma^{n+1} r_{0}\right)}>\frac{n \log \left\{\frac{1}{c(2-c)}\right\}+\log T\left(r_{0}\right)}{(n+1) \log \sigma+\log r_{0}}, \\
\mu=\liminf _{r \rightarrow \infty} \frac{\log T(r)}{\log r} \geqq \frac{\log \left\{\frac{1}{c(2-c)}\right\}}{\log \sigma} .
\end{gathered}
$$

Theorem 4 now follows by letting $c \rightarrow \gamma$.

10. Proof of Theorem 5. In this section, we put

$$
\sigma=(35)^{1 / \beta}, \quad \rho=\frac{r}{\alpha \sigma}, \quad R=\frac{\sigma r}{\alpha} .
$$

We show first that, in view of (2.11), 


$$
\begin{aligned}
2 T(r)-2 N(r) & <(q+1) r^{q} \int_{\rho}^{R} N(\alpha t) t^{-q-1} \phi\left(\frac{t}{r}\right) d t \\
& +8.5\left(\frac{r}{\rho}\right)^{q} T(\alpha \rho)+8.5\left(\frac{r}{R}\right)^{q+1} T(\alpha R) \quad(q \geqq 0),
\end{aligned}
$$

provided $r$ is sufficiently large.

If $f(0)=1$, this follows inmediately from $(2.10),(2.9)$ and the inequality

$$
N(u)<\frac{1}{27} T(u)
$$

which is a consequence of (2.11), for all large $u$.

In fact the constants 8.5 could be replaced by the smaller number $8+10 / 27$. If $f(0) \neq 1$, we replace $f(z)$ by

$$
f_{1}(z)=A z^{\nu} f(z),
$$

where the constant $A$ and the integer $\nu$ are determined so that $f_{1}(0)=1$. The functions $N_{1}$ and $T_{1}$ associated with $f_{1}$, in the same way that $N$ and $T$ are associated with $f$, satisfy

$$
\begin{aligned}
N_{1}(u) & \leqq N(u) \\
T_{1}(u) & =T(u)+O(\log u)
\end{aligned}
$$$$
\begin{array}{r}
(u \geqq 1), \\
(u \rightarrow+\infty) .
\end{array}
$$

Since $f(z)$ and $f_{1}(z)$ obviously are not rational functions,

$$
\frac{\log u}{T(u)} \rightarrow 0
$$

as $u \rightarrow+\infty$ and so, by our choice of $\rho$,

as $r \rightarrow+\infty$.

$$
\frac{\log r}{T(\alpha \rho)} \rightarrow 0
$$

Now (10.2) holds with $N$ and $T$ replaced by $N_{1}$ and $T_{1}$. In view of (10.3) and (10.4), it is possible to return to $N$ and $T$ provided suitable logarithmic error terms are introduced. These terms may be absorbed in the term with $T(\alpha \rho)$, at the expense of the increase in the numerical factor from $8+10 / 27$ to 8.5 .

Next we prove

LEMMA 3. If (2.11) holds and if

$$
\begin{aligned}
\sigma & =(35)^{1 / \beta}, \\
q+\beta & \leqq c \leqq q+1-\beta,
\end{aligned}
$$

then, 
1959] MEROMORPHIC FUNCTIONS WITH SEVERAL DEFICIENT VALUES 319

$$
\frac{T(r)}{r^{c}}<\sup _{r / \sigma \leqq u \leqq \sigma r} \frac{T(u)}{u^{c}}
$$

provided $r$ exceeds a suitable positive bound $r_{0}$.

Proof. Put

$$
\tau=\frac{\beta}{5 e(q+1)}
$$

so that, by (2.11),

$$
N(u)<\tau T(u)
$$

for all large $u$.

Suppose now that $r$ is so large that (10.2) holds and that (10.7) is valid for all $u>(r / \sigma)$. Suppose also that $(10.6)$ is violated, so that

$$
T(u) \leqq\left(\frac{u}{r}\right)^{c} T(r) \quad\left(\frac{r}{\sigma} \leqq u \leqq \sigma r\right) .
$$

Then, by (10.2), (10.1), (10.7) and (10.8)

$$
\begin{aligned}
(2-2 \tau) T(r)= & (q+1) \tau T(r) r^{q} \int_{\rho}^{R}\left(\frac{\alpha t}{r}\right)^{c} t^{-q-1} \phi\left(\frac{t}{r}\right) d t+8.5(\alpha \sigma)^{q} T(r) \sigma^{-c} \\
& +8.5\left(\frac{\alpha}{\sigma}\right)^{q+1} T(r) \sigma^{c} .
\end{aligned}
$$

Hence, dividing by $T(r)$ and putting $v=t / r$, we obtain

$$
2-2 \tau \leqq(q+1) \tau \alpha^{c} \int_{(\rho / r)}^{(R / r)} v^{c-q-1} \phi(v) d v+8.5 \alpha^{q} \sigma^{q-c}
$$

$$
+8.5 \alpha^{q+1} \sigma^{c-q-1} \text {. }
$$

Let $J$ denote the integral in (10.9); by (3.13) and (10.5),

$$
J<\int_{0}^{\infty} v^{c-q-1} \phi(v) d v<\frac{4.4}{\sin \pi(c-q)}<\frac{4.4}{\sin \pi \beta}<\frac{2.2}{\beta} .
$$

We finally notice that, in view or (10.5), the definitions of $\alpha$ and $\sigma$ imply

$$
\begin{gathered}
\alpha^{q}<\alpha^{c}<\alpha^{q+1}=e, \\
\sigma^{q-c} \leqq \sigma^{-\beta}, \quad \sigma^{c-q-1} \leqq \sigma^{-\beta}=\frac{1}{35} .
\end{gathered}
$$

Using the estimates (10.10), (10.11) and (10.12), in (10.9), we obtain

$$
2 \leqq 2 \tau+\frac{2.2 e(q+1) \tau}{\beta}+\frac{17 e}{35},
$$


and in view of the definition of $\tau$,

$$
2 \leqq \frac{1}{5 e}+\frac{2.2}{5}+\frac{48}{35}<2 .
$$

This contradiction, which is a consequence of (10.8), completes the proof of Lemma 3.

We now prove assertion II of Theorem 5. Let

$$
c=q+\beta \text {. }
$$

By assumption (2.14), $c>\mu$ and hence

$$
\liminf _{u \rightarrow \infty} \frac{T(u)}{u^{c}}=0 .
$$

We first show that (10.13) implies the following property: Given $u_{0}(>0)$ it is possible to find $y_{0}\left(>u_{0}\right)$ such that

$$
\frac{T\left(y_{0}\right)}{y_{0}^{c}}=\sup _{\nu_{0} \leqq u \leqq \sigma_{0}} \frac{T(u)}{u^{c}} .
$$

In view of (10.13), there exists some $v\left(>u_{0}\right)$ such that

$$
\sigma^{c} \frac{T(v)}{v^{c}}<\frac{T\left(u_{0}\right)}{u_{0}^{c}} .
$$

At some point $y$ of the closed interval $\left[u_{0}, v\right]$

$$
\frac{T(y)}{y^{c}}=\sup _{u_{0} \leqq u \leqq v} \frac{T(u)}{u^{c}} .
$$

This definition of $y$ and (10.15) imply

$$
\frac{T(y)}{y^{c}}>T(v)\left(\frac{\sigma}{v}\right)^{c} \text {. }
$$

If

$$
v \leqq \sigma y,
$$

(10.17) yields

$$
\frac{T(y)}{y^{c}}>\frac{T(v)}{y^{c}} \geqq \frac{T(y)}{y^{c}} .
$$

This contradiction shows that (10.18) is impossible; therefore

$$
u_{0} \leqq y<\sigma y<v \text {, }
$$

and hence, by (10.16), 


$$
\frac{T(y)}{y^{c}}=\sup _{\nu \leqq u \leqq \sigma \nu} \frac{T(u)}{u^{c}} .
$$

Taking $y_{0}=y$, we obtain (10.14).

It is now clear that we may find an increasing, unbounded sequence $\left\{y_{v}\right\}_{v=1}^{\infty}$, such that

$$
\frac{T\left(y_{v}\right)}{y_{v}^{c}}=\sup _{v_{\nu} \leqq u \leqq \sigma \nu_{v}} \frac{T(u)}{u^{c}}
$$

It is also possible to assume

$$
y_{1}>r_{0}
$$

where $r_{0}$ is the bound in Lemma 3 .

We now associate, with each $y_{v}$, a sequence $Y_{v}$ containing a finite number of elements:

$$
t_{0}^{(v)}, t_{1}^{(v)}, \cdots, t_{j}^{(v)}
$$$$
(j=j(\nu)) .
$$
$t_{k}^{(\nu)}$.

Since our arguments involve a single sequence $Y_{\nu}$, we write $t_{k}$ instead of DEFinition OF $Y_{\nu}$.

(i) Let

$$
t_{0}=y_{\nu}
$$

and take $t_{1}$ to be some point of the closed interval $\left[t_{0} / \sigma, \sigma t_{0}\right]$ such that

$$
\frac{T\left(t_{1}\right)}{t_{1}^{c}}=\sup _{t_{\alpha} \leqq u \leqq \sigma t_{0}} \frac{T(u)}{u^{c}} .
$$

By assumption,

$$
t_{0} \geqq y_{1},
$$

so that, in view of $(10.20)$, we may apply Lemma 3 with $r=t_{0}$. This yields

$$
\frac{T\left(t_{1}\right)}{t_{1}^{c}}>\frac{T\left(t_{0}\right)}{t_{0}^{c}} \text {. }
$$

Since by (10.19)

$$
\frac{T\left(t_{0}\right)}{t_{0}^{c}}=\sup _{t_{0} \leqq u \leqq t_{0}} \frac{T(u)}{u^{c}}
$$

it is clear that

$$
\frac{t_{0}}{\sigma} \leqq t_{1}<t_{0}
$$


and also

$$
\frac{T\left(t_{1}\right)}{t_{1}^{c}}=\sup _{t_{1} \leqq u \leqq \sigma t_{1}} \frac{T(u)}{u^{c}} .
$$

(ii) Notice that (10.25) is the same relation as (10.23) with $t_{0}$ replaced by $t_{1}$. Hence the arguments which lead from $t_{0}$ to $t_{1}$ may be repeated with $t_{0}$ replaced by $t_{1}$, provided Lemma 3 is still applicable. This requires $r_{0}<t_{1}$. If $t_{1} \leqq r_{0}$, we interrupt our construction and take $Y_{\nu}$ to be the sequence: $t_{0}, t_{1}$. Otherwise, we define successively $t_{2}, t_{3}, \cdots, t_{j}$ and terminate $Y_{v}$ with $t_{j}$ characterized by

$$
t_{j} \leqq r_{0}<t_{j-1} .
$$

It is clear, by our construction of $Y_{v}$, that the relations (10.21), (10.22), (10.23) and (10.24) imply

$$
\frac{T\left(t_{k+1}\right)}{t_{k+1}^{c}}>\frac{T\left(t_{k}\right)}{t_{k}^{c}} \geqq \frac{T(u)}{u^{c}} \quad\left(t_{k} \leqq u \leqq t_{0} ; k=0,1, \cdots, j-1\right),
$$

and

$$
\frac{t_{k}}{\sigma} \leqq t_{k+1}<t_{k} \quad(k=0,1, \cdots, j-1) .
$$

(iii) In order to see that $Y_{\nu}$ has a finite number of terms, we prove

$$
t_{k+1}<\frac{t_{k-1}}{\sigma}
$$

If this inequality were false, we would have

$$
\frac{T\left(t_{k+1}\right)}{t_{k+1}^{c}} \leqq \sup _{t_{k-1} / \sigma \leqq u \leqq \sigma t_{k-1}} \frac{T(u)}{u^{c}}=\frac{T\left(t_{k}\right)}{t_{k}^{c}},
$$

which contradicts the first of the inequalities (10.27).

Now let $x\left(>r_{0}\right)$ be given and choose some point $t$, in the closed interval $[x, \sigma x]$, where

$$
\frac{T(t)}{t^{c}}=\sup _{x \leqq u \leqq \sigma x} \frac{T(u)}{u^{c}} .
$$

Given $v(\geqq t)$, we select some $y_{v}$ such that

$$
v<y_{v}, \quad \sigma x<y_{v} .
$$

Then

$$
t_{j} \leqq r_{0}<x<\sigma x<t_{0},
$$


and, in view of (10.28), some member $t_{k}$, of $Y_{\nu}$, falls in the closed interval $[x, \sigma x]$. Hence

$$
\frac{T(t)}{t^{c}} \geqq \frac{T\left(t_{k}\right)}{t_{k}^{c}}
$$

If

$$
t \leqq v \leqq \sigma x,
$$

the inequality

$$
\frac{T(t)}{t^{c}} \geqq \frac{T(v)}{v^{c}},
$$

follows from (10.29).

If $\sigma x<v$, then

$$
t_{k}<v
$$

and, by the first inequality $(10.30)$

$$
v<t_{0} .
$$

In this case, (10.32) follows from (10.31) and the second inequality (10.27). This completes the proof of assertion II of Theorem 5.

The proof of assertion I is along similar lines, but simpler in detail.

Put

$$
c=q+1-\beta
$$

and let $r_{0}(>0)$ be the bound in Lemma 3 .

The assumption $\lambda>c$ implies

$$
\limsup _{u \rightarrow \infty} \frac{T(u)}{u^{c}}=+\infty,
$$

and hence, it is possible to find a point $u_{0}\left(>\sigma r_{0}\right)$ such that

$$
\frac{T\left(u_{0}\right)}{u_{0}^{c}}>\sup _{r_{0} \leqq u \leqq \sigma r_{0}} \frac{T(u)}{u^{c}},
$$

and then a point $s_{1}$, of the closed interval $\left[r_{0}, u_{0}\right]$, such that

$$
\frac{T\left(s_{1}\right)}{s_{1}^{c}}=\sup _{r 0 \leqq u \leqq u_{0}} \frac{T(u)}{u^{c}} .
$$

By (10.33) and (10.34), it is clear that

$$
\sigma r_{0}<s_{1},
$$

and also that 


$$
\frac{T\left(s_{1}\right)}{s_{1}^{c}}=\sup _{r_{0} \leqq u \leqq s_{1}} \frac{T(u)}{u^{c}} .
$$

We next choose some point $s_{2}$ belonging to $\left[s_{1} / \sigma, \sigma s_{1}\right]$ and such that

$$
\frac{T\left(s_{2}\right)}{s_{2}^{c}}=\sup _{s_{1} / \sigma \leqq u \leqq \sigma s_{1}} \frac{T(u)}{u^{c}} .
$$

Since $s_{1}>r_{0}$, Lemma 3 yields

$$
\frac{T\left(s_{2}\right)}{s_{2}^{c}}>\frac{T\left(s_{1}\right)}{s_{1}^{c}}
$$

and in view of (10.35)

$$
s_{1}<s_{2} \leqq \sigma s_{1} .
$$

It is also clear, by (10.38), (10.36), (10.37) and (10.35), that

$$
\frac{T\left(s_{2}\right)}{s_{2}^{c}} \geqq \frac{T(u)}{u^{c}} \quad\left(r_{0} \leqq u \leqq s_{2}\right) .
$$

Repeated application of the same construction leads to a sequence $\left\{s_{k}\right\}_{k=1}^{\infty}$ such that

$$
s_{k}<s_{k+1} \leqq \sigma s_{k} \quad(k=1,2,3, \cdots),
$$

and

$$
\frac{T\left(s_{k+1}\right)}{s_{k+1}^{c}}>\frac{T\left(s_{k}\right)}{s_{k}^{c}} \geqq \frac{T(u)}{u^{c}} \quad\left(r_{0} \leqq u \leqq s_{k}, k=1,2,3, \cdots\right) .
$$

In order to see that the sequence $\left\{s_{k}\right\}_{k=1}^{\infty}$ is not bounded, we prove

$$
\sigma s_{k-1}<s_{k+1} \text {. }
$$

If this inequality were not true, we would have

$$
\frac{T\left(s_{k+1}\right)}{s_{k+1}} \leqq \sup _{\delta_{k-1} / \sigma \leqq u \leqq \sigma s_{k-1}} \frac{T(u)}{u^{c}}=\frac{T\left(s_{k}\right)}{s_{k}^{c}},
$$

which contradicts the first of the inequalities (10.40).

In view of (10.39), it is clear that, if $x>s_{1}$, each closed interval $[x, \sigma x]$ contains a point $s$ of the sequence $\left\{s_{k}\right\}_{k-1}^{\infty}$. Assertion I of Theorem 5 now follows from the second of the inequalities (10.40).

11. Proof of Corollary 5.1. If (2.11) and (2.12) are satisfied, assertion I of Theorem 5 enables us to associate, with each $r\left(>x_{0} \sigma\right)$ an $s$, in $[r / \sigma, r]$, such that 


$$
T(r) \geqq T(s) \geqq T\left(x_{0}\right)\left(\frac{s}{x_{0}}\right)^{q+1-\beta} \geqq T\left(x_{0}\right)\left(\frac{r}{\sigma x_{0}}\right)^{q+1-\beta}
$$

Hence

$$
\mu \geqq q+1-\beta .
$$

Similarly, if (2.11) and (2.14) are satisfied, there will exist, by assertion II of Theorem 5, some $t$ such that $r \geqq t$ implies

$$
T(r) \leqq\left(\frac{r}{t}\right)^{q+\beta} T(t) .
$$

Hence

$$
\lambda \leqq q+\beta .
$$

12. Proof of Theorem 6 . We show first that $p \geqq 1$.

Since by the definition of $\kappa(f)$ and (2.15),

$$
\gamma=\max \{1-\delta(0), 1-\delta(\infty)\}<\kappa(f)<\frac{1}{10 e},
$$

Theorem 4 gives $\mu=1$ if $\gamma=0$, and

$$
\mu \geqq \frac{\log \left\{\frac{1}{\gamma(2-\gamma)}\right\}}{\log \left(1+\frac{4}{\gamma(1-\gamma)}\right)}>\frac{\log \left(\frac{1}{2 \gamma}\right)}{\log \left(\frac{1}{2 \gamma}\right)+\log \left\{\frac{8}{1-\gamma}+2 \gamma\right\}}
$$

if $\gamma \neq 0$. By (12.1)

$$
10<5 e<\frac{1}{2 \gamma}, \quad \frac{1}{1-\gamma}<\frac{10}{9}
$$

and hence

$$
\frac{8}{1-\gamma}+2 \gamma<\frac{80}{9}+\frac{1}{10}<10<\frac{1}{2 \gamma} .
$$

It is now clear that (12.2) implies $\mu>1 / 2$ and therefore $p \geqq 1$.

We next show that

$$
\lambda \leqq p+1-\beta .
$$

If this inequality were false, we could, in view of (2.15), apply Corollary 5.1 with $q=p$ and obtain

$$
\mu \geqq p+1-\beta,
$$


contrary to hypothesis. By (2.15), Theorem 1 and (12.3)

$$
\frac{\beta}{5 e(p+1)}>\kappa(f) \geqq \frac{|\sin \pi \lambda|}{2.2 \lambda+\frac{1}{2}} \geqq \frac{|\sin \pi \lambda|}{2.2(p+1-\beta)+\frac{1}{2}} \geqq \frac{|\sin \pi \lambda|}{e(p+1)} .
$$

Hence

$$
|\sin \pi \lambda|<\frac{\beta}{5} .
$$

If $l$ is one of the integers closest to $\lambda$, then

$$
2|l-\lambda| \leqq|\sin \pi(l-\lambda)|=|\sin \pi \lambda|<\frac{\beta}{5} .
$$

Since

$$
p-(1 / 2) \leqq \mu \leqq \lambda \leqq p+1-\beta,
$$

this leaves only the possibility $l=p$,

$$
|\lambda-p|<\frac{\beta}{10} \text {. }
$$

The last unproved assertion of the theorem, $\mu \geqq p-\beta$, now follows from Corollary 5.1 with $q+1=p$.

13. Proof of Corollary 6.1. Suppose first that $f(z)$ is of infinite order. Then (2.11) and (2.12) hold for every integer $q(\geqq 0)$ and, by Corollary $5.1, \mu$ is greater than any assigned integer. Hence $\mu=+\infty$ and the result is proved for functions of infinite order.

If $\lambda<+\infty$, then Corollary 6.1 follows at once from Theorem 6 .

14. Proof of Lemma 1. The following inequalities are implicit in R. Nevanlinna's proof of the second Fundamental Theorem [5, p. 64]. They were stated explicitly by Ullrich [7, p. 207].

For every meromorphic function $f(z)$, of finite order, and every finite set of complex numbers $\tau_{1}, \tau_{2}, \cdots, \tau_{p}$

$$
\begin{aligned}
\sum_{v=1}^{p} m\left(r, \frac{1}{f-\tau_{\nu}}\right)+N\left(r, \frac{1}{f^{\prime}}\right)+O(\log r) & \leqq T\left(r, f^{\prime}\right) \\
& \leqq m(r, f)+N\left(r, f^{\prime}\right)+O(\log r) .
\end{aligned}
$$

Since $f^{\prime}(z)$ has a pole of order $k+1$ where $f(z)$ has a pole of order $k$

$$
N\left(r, f^{\prime}\right) \leqq 2 N(r, f) \text {. }
$$

For $\epsilon>0$ and all sufficiently large $r$,

$$
N(r, f)<(1-\delta(\infty, f)+\epsilon) T(r, f) .
$$


Using (14.2) and (14.3) in the second of the inequalities (14.1), we obtain

$$
T\left(r, f^{\prime}\right) \leqq T(r, f)+(1-\delta(\infty, f)+\epsilon) T(r, f)+O(\log r),
$$

and in view of $(2.17)$,

$$
\limsup _{r \rightarrow \infty} \frac{T\left(r, f^{\prime}\right)}{T(r, f)} \leqq 2-\delta(\infty, f)<1+\gamma .
$$

The first of the inequalities (14.1) yields

$$
\begin{aligned}
\underset{r \rightarrow \infty}{\liminf } \frac{T\left(r, f^{\prime}\right)}{T(r, f)} & \geqq \liminf _{r \rightarrow \infty} \sum_{r=1}^{p} \frac{m\left(r, \frac{1}{f-\tau_{v}}\right)}{T(r, f)} \\
& \geqq \sum_{v=1}^{p} \liminf _{r \rightarrow \infty} \frac{m\left(r, \frac{1}{f-\tau_{v}}\right)}{T(r, f)}=\sum_{r=1}^{p} \delta\left(\tau_{v}, f\right) .
\end{aligned}
$$

Since $p$ is arbitrary, (14.5) and (2.16) imply

$$
\liminf _{r \rightarrow \infty} \frac{T\left(r, f^{\prime}\right)}{T(r, f)} \geqq \Delta>1-\gamma .
$$

Combining (14.4) and (14.6), we obtain (2.18).

Now by (14.2), (14.6), (2.16) and (2.17)

$$
\begin{aligned}
\underset{r \rightarrow \infty}{\limsup } \frac{N\left(r, f^{\prime}\right)}{T\left(r, f^{\prime}\right)} & \leqq 2 \limsup _{r \rightarrow \infty}\left\{\frac{N(r, f)}{T(r, f)} \cdot \frac{T(r, f)}{T\left(r, f^{\prime}\right)}\right\} \\
& \leqq 2(1-\delta(\infty, f)) \frac{1}{\Delta}<\frac{2 \gamma}{1-\gamma}
\end{aligned}
$$

The first inequality (14.1) may be rewritten in the form

$$
\frac{N\left(r, \frac{1}{f^{\prime}}\right)}{T\left(r, f^{\prime}\right)}+\frac{T(r, f)}{T\left(r, f^{\prime}\right)} \sum_{r=1}^{p} \frac{m\left(r, \frac{1}{f-\tau_{\nu}}\right)}{T(r, f)}+O\left(\frac{\log r}{T\left(r, f^{\prime}\right)}\right) \leqq 1 .
$$

Hence

$$
\limsup _{r \rightarrow \infty} \frac{N\left(r, \frac{1}{f^{\prime}}\right)}{T\left(r, f^{\prime}\right)} \leqq 1-\liminf _{r \rightarrow \infty} \frac{T(r, f)}{T\left(r, f^{\prime}\right)} \sum_{r=1}^{p} \liminf _{r \rightarrow \infty} \frac{m\left(r, \frac{1}{f-\tau_{p}}\right)}{T(r, f)},
$$

and in view of (14.4) 


$$
\limsup _{r \rightarrow \infty} \frac{N\left(r, \frac{1}{f^{\prime}}\right)}{T\left(r, f^{\prime}\right)} \leqq 1-\frac{\sum_{\nu=1}^{p} \delta\left(\tau_{\nu}, f\right)}{1+\gamma} .
$$

Since $p$ is arbitrary, this inequality implies

$$
\limsup _{r \rightarrow \infty} \frac{N\left(r, \frac{1}{f^{\prime}}\right)}{T\left(r, f^{\prime}\right)} \leqq 1-\frac{\Delta}{1+r} .
$$

Using (14.7), (14.8) and (2.16), we finally obtain

$\kappa\left(f^{\prime}\right) \leqq \limsup _{r \rightarrow \infty} \frac{N\left(r, f^{\prime}\right)}{T\left(r, f^{\prime}\right)}+\limsup _{r \rightarrow \infty} \frac{N\left(r, \frac{1}{f^{\prime}}\right)}{T\left(r, f^{\prime}\right)} \leqq \frac{2 \gamma}{1-\gamma}+1-\frac{1-\gamma}{1+\gamma}=\frac{4 \gamma}{1-\gamma^{2}}$.

This completes the proof of Lemma 1.

\section{REFERENCES}

1. A. Edrei and W. H. J. Fuchs, Valeurs deficientes et valeurs asymptotiques des fonctions méromorphes, to appear in Comment. Math. Helv.

2. - On the deficiencies of meromorphic functions of order less than one, to appear in Duke Math. J.

3. A. A. Goldberg, On the deficiencies of meromorphic functions (in Russian), Dokl. Akad. Nauk. USSR vol. 98 (1954) pp. 893-895.

4. F. Nevanlinna, Ueber eine Klasse meromorpher Funktionen, 7 ème Congr. Math. Scand. Oslo, vol. 7, 1930, pp. 81-83.

5. R. Nevanlinna, Le theorème de Picard-Borel et la théorie des fonctions méromorphes, Paris, 1930.

6. E. C. Titchmarsh, The theory of functions, 2d ed., Oxford, 1950.

7. E. Ullrich, Ueber den Einfluss der Verzweigtheit einer Algebroide auf ihre Wertverteilung, J. Reine Angew. Math. vol. 167 (1932) pp. 198-220.

8. G. Valiron, Sur les fonctions entières d'ordre fini et d'ordre nul, et en particulier les fonctions a correspondance régulière, Ann. Fac. Sci. Univ. Toulouse vol. 5 (3) (1913) pp. 117-257.

9. - Lectures on the general theory of integral functions, Toulouse, 1923.

10. - Valeurs exceptionnelles et valeurs déficientes des fonctions méromorphes, C. R. Acad. Sci. Paris vol. 225 (1947) pp. 556-558.

11. E. T. Whittaker and G. N. Watson, $A$ course of modern analysis, 4 th ed., Cambridge, 1940.

SyRACUSE UNIVERSITY, Syracuse, NEW YORK

CORNELL UNIVERSITY, ITHACA, NEW YORK 